\title{
Power Demand Side Management And Demand Response
}

\author{
Su Xinxia ${ }^{1, a}$, Wang Zhijie ${ }^{1, b}$, Ye Yizhi ${ }^{2, c}$, Chen Lijuan $^{1, d}$, Pei Zeyang ${ }^{1, e}$ \\ ${ }^{1}$ College of Electrical Engineering, Shanghai DianJi University, China, 200240 \\ ${ }^{2}$ Shanghai Electric Center Academe, Shanghai Electric, China, 200070 \\ asuxinxia1991@126.com, bwzjsdstu@163.com, cyeyz@shanghai-electric.com \\ dchenlijuancom@163.com, eyangyang0818ab@163.com
}

Keywords: power demand side management; demand response; demand response measures

\begin{abstract}
This paper first introduces the concept of demand side management and its relationship with demand response; and then introduces problems and solutions involved in the process of implying the demand side management, such as the ladder electricity price, classify the electricity users, predict the loads and evaluate the benefits; finally introduces the concept and classification of demand response, and has made the concrete interpretation for each response measures.
\end{abstract}

\section{Introduction}

The demand side has been considered to be pure electric power consumption unit for a long time. Until 1970s' energy crisis, some countries are no longer blindly pursuit of the installed capacity to meet the load demands, but through improve the unit's use efficiency and cut loads to solve the contradiction between supply and demand. Power Demand Side Management (DSM) are management activities refer to the power companies to adopt effective incentive and induced measures and suitable operation way, cooperate with users to enhance the end use efficiency, reduce the power consumption and power demand. It mainly include the power load regulation, energy conservation, and novel energy substitution and application aspects.

The original intention of Demand Response (DR) is to encourage consumers to change the use of electricity during peak load period through rational designed mechanism, and discontinued or transferred use to the bottom period. Thus reducing peak load is this primary objective. The mode of its actions are default and the action time often limited to peak load period. As a result, the function this demand response can played in power system dispatching is limited. When go to the consumption of wind power, it is clearly that demand side should response obey the system operation needs. Because they are enable frequently, the load must be a kind of non-time sensitive loads, such as electric cars, refrigeration and heating equipment with energy storage characteristics.

\section{Demand side management}

DSM refers to the power supply and demand both sides jointly manage the electricity market, to improve power supply reliability, reduce energy consumption and the purpose of the supply and demand both sides expense. Its content includes load control and management and the distance meter reading and billing automation. Power DSM is dominated by the government, with power companies as the main implement promotion units, aimed at economic incentives as the main means, guide and stimulate the general electric power users optimize utilization way, improve the efficiency of terminal power, achieve significant power saving electric power systems engineering.

At present, China should incorporate DSM in energy development and overall planning for economic and social development of a unified, unified deployment. Perfecting the relevant laws and regulations and establish long-term effective mechanism of demand side management. Formulate relevant policies and measures to raise the necessary funds, actively support demand side management. Study on electricity price linkage mechanism, through the economic lever to adjust 
electricity demand. The development of new technology, vigorously to adopt new equipment, provide technical support for DSM.

\section{How to establish the ladder electricity price}

Ladder electricity price basis theory is Ramsey pricing strategy, and its core idea is maximizing social welfare under the restriction of budget balance through pricing model "inversely proportional to the elastic". Because the residents electricity elasticity of demands is closely related to income levels, the ladder electricity price is according to different income groups for different price, whereas in the practice usually according to different power consumption segmentation. However, people's income, monthly electricity consumption as well as the relationship between the two are difficult to accurately grasp. So the above principle in practice is not easy, it need to use relevant information to determine. Based on the Keynes consumption function, it determine the relationship between the residents' monthly electricity consumption and their income, and then according to the residents income statistics to determine the power and electricity prices. Ladder electricity price is formulate with the user power consumption structure, level, residents' income and other factors, so the current research remains to be further.

\section{How to classify the electricity users}

The classification of the electric power users usually based on some obvious contract characteristics, such as the properties of electricity, electricity consumption, access voltage grades and so on. The classification as more incentive users' clustering analysis problem, we can try a variety of clustering algorithms from the method based on distance to the Support Vector Machine (SVM) method. Clustering analysis is an important branch of data mining, and mainly divided into the classification methods and clustering methods. Classification methods include classification method based on distance, decision tree classification method, Bayesian classification method and rule induction classification method. Clustering methods are divided into the hierarchical clustering algorithm, hierarchical clustering algorithm, density clustering algorithm, and other clustering algorithms.

\section{How to predict the loads}

Electric power marketing department has accumulated massive users' power-expenditure information datum, and it contain abundant laws about loads information. It is an effective means to reveal the law of loads by proceeding regression analysis to this datum. SVM is a kind of new machine learning theory in data mining technology, and it get very good application in power system load forecasting. Linear regression method, statistical regression algorithm method in other words, is simple. However, SVM regression has a better sample adaptability, and has unique advantages in the regression analysis of nonlinear and high dimensional pattern. So generally we use SVM regression analysis to predict the loads.

\section{How to evaluate the benefits}

Demand side management evaluation should focus on the main characteristics of the volume, including response capacity, response speed, frequency, load recovery time and so on. These characteristics are key concerns for demand response behavior modeling. Evaluate the demand side management value should consider the interests of the generator side, grid side, power users as well as the whole society benefits (including the influence of demand side response to the environment and the effective use of resources). In determining the index weight we need to select a method of strong objectivity to maximize reduce the subjectivity of evaluation results. Entropy weight method is a kind of objective empowerment. It based on the analysis of the trend of the indexes, determining the entropy value of each index by means of information entropy, and then using the entropy weight correction of each index weight, thus draws the objective index weight. Technique for Order Preference by Similarity to an Ideal Solution (TOPSIS) method evaluation objects to sort with the help of positive and negative ideal solution of the evaluation objects. It has been used in risk assessment, multi-objective optimization, power grid planning and many other fields. So, in general, entropy weight method combined with TOPSIS method can be used to do demand side management efficiency evaluation. 


\section{Definition of demand response}

DR refers to the users of power market change the conventional power consumption mode and change their market participation behavior according to market price signals or incentive mechanisms from the broad sense. From the perspective of resources, DR can be used as a kind of resources, and by the reduction of the peak loads or installed capacity. From the perspective of ability, DR can improve the operation reliability of power grid and enhance power grid emergency capability. From the perspective of behavior, DR refers to the user participate in load management through adjusting power using mode.

Taking the above different angles and the actual situation in China into consideration, DR refers to under different degrees of marketization, through technical, economic, administrative, legal and other means to encourage and guide users voluntary to change the conventional electric using methods and then use electricity scientifically and rationally, with the aim of promoting the optimal allocation of power resources and guaranteeing the power system operate safely and economically.

\section{Demand response measures}

Demand response measures can be divided into two main types: price based demand response measures and incentive based demand response measures. Price based demand response means take electricity price as incentives to encourage users to adjust the electricity consumption according to different prices. It include Time of Use Pricing (TOU), Critical Peak Pricing (CPP) and Real Time Pricing (RTP). Incentive based demand response means that the project implement agencies promote the users to participate in the plan that shedding loads when electricity price is higher or the reliability of the system is affected, through the establishment of some economic incentives or compensation mechanism. It include Direct Load Control (DLC), Interruptible Load (IL), Demand Side Bidding (DSB), Emergency Demand Response Program (EDRP) and Capacity/Ancillary Service Program (CASP).

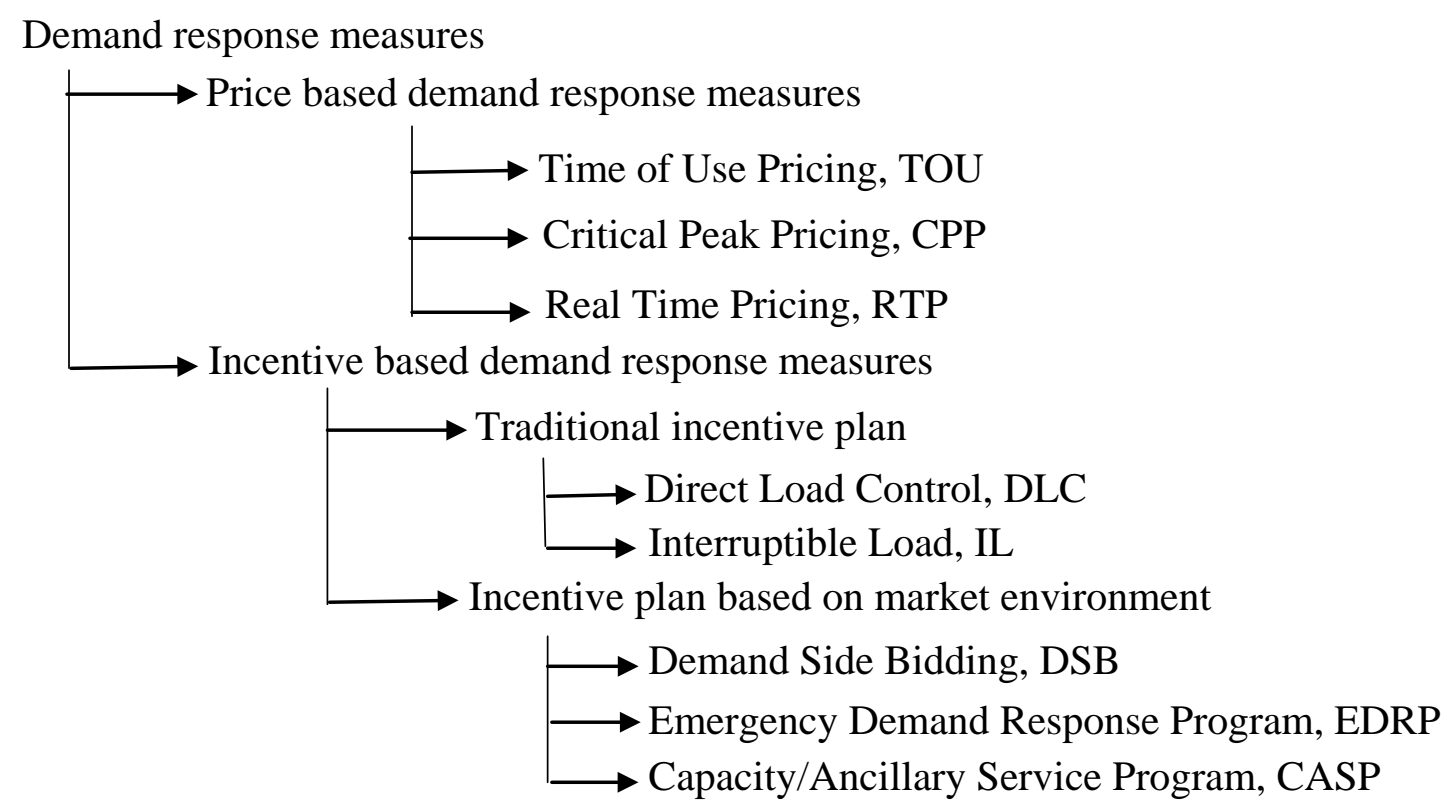

Fig. 1 demand response measures 
Here I will introduce the concrete implementing schemes of different types of demand response shown in figure 1.

TOU pricing is a mechanism that effectively can reflect difference power system supply cost between different periods. Peak-valley electricity price, season-of-use price and abundant-dry water price are the most common forms of it. An improvement form of this is load selection. The participators can select load curtailment, cut time and advance notice time according to different price levels in different load time. The price levels are associated with the advance notice time. The shorter the time, the higher the price.

RTP is a dynamic pricing mechanism and the update cycle can reach 1 hours or less. The user side price and the electricity supply market clearing price linkage can reflect the changes in each period of the day supply cost and effectively convey the price signal. RTP make up for lacking of incentive in TOU when the system capacity is shortage and do not able to further cut loads.

CPP is a dynamic pricing mechanism in foundation of TOU and RTP. Its main idea is add peak price on the time of use price. Implementing agencies previously published peak event time while set standards and the corresponding peak rate. Price in non-peak period execute TOU and execute peak rate in peak period with notifying the user ahead of a certain time (usually 1 days). Users can make the corresponding electricity plan adjustment through advanced meter.

DLC means direct load control mechanism can close or control electrical equipment of users through the remote control device in peak hours.

IL is based on the contract made by supply and demand sides in advance, sending interrupt request signal to user of interruptible load by implementing agencies in power grid peak time, and interrupt those power after the user response. For users require electricity reliability is not so high, they can reduce or stop a part of electricity to avoid the grid peak, and can obtain the corresponding interrupt compensation. Interruptible load is generally applicable to large industrial and commercial users. It is an ideal control method for the grid peak load shifting.

DSB is an implementation mechanism that demand side resources participate in electricity market competition. It enables users actively take part in market competition and get the corresponding economic interests by changing their way of using electricity, rather than are price recipient purely. Power supply companies and large users can directly participate in demand side bidding, while small scattered users can indirectly participate in it through comprehensive load agency.

EDRP refers to a way that user response and interrupt electric power demand in response to the sudden case of emergency and according to the power grid load adjustment requirement and the price levels. It combines the history data, price data and short-term load forecasting. It is used to cut the peak load and avoid the peak price.

CASP means the users provide cutting load as the backup system replacing traditional generators or providing a form of resources.

\section{Project support}

This paper is funded by Shanghai municipal education commission key projects of scientific research innovation (12 zz197), Shanghai natural science fund (12 zr1411600), Shanghai science and technology innovation project (2011 mh065/2011 mh097 mh089/2011/2011 mh099), national natural science foundation of China (11201267) projects.

\section{References}

[1] Wu, C., Mohsenian-Rad, H., Huang, J., \& Wang, A. Y. (2011, December). In GLOBECOM Workshops (GC Wkshps), 2011 IEEE (pp. 1199-1204). IEEE.

[2] Moura, P. S., \& De Almeida, A. T. (2010). Applied Energy, 87(8), 2581-2588.

[3] Prüggler, N., Prüggler, W., \& Wirl, F. (2011). Energy, 36(11), 6308-6317. 
[4] Spiegel, R. J., Kern Jr, E. C., \& Greenberg, D. L. (1998). Solar Energy, 62(5), 345-358.

[5] Affonso, C. M., da Silva, L. C. P., \& Freitas, W. (2006, May). In Transmission and Distribution Conference and Exhibition, 2005/2006 IEEE PES (pp. 517-522). IEEE. 